EPJ manuscript No.

(will be inserted by the editor)

\title{
Neutron-proton mass difference in finite nuclei and the Nolen-Schiffer anomaly
}

\author{
Ulf-G. Meißner ${ }^{1,2}$, A. M. Rakhimov ${ }^{3,4}$, A. Wirzba ${ }^{2}$, and U. T. Yakhshiev ${ }^{2,5}$ \\ 1 Helmholtz-Institut für Strahlen- und Kernphysik (Theorie), D-53115, Universität Bonn, Germany \\ 2 Forschungszentrum Jülich, Institut für Kernphysik (Theorie), D-52425 Jülich, Germany \\ 3 Institute of Nuclear Physics, Academy of Sciences of Uzbekistan, Tashkent-132, Uzbekistan \\ 4 Institute of Physics and Applied Physics, Yonsei University, Seoul, 120-749, Korea \\ 5 Physics Department and Institute of Applied Physics, National University of Uzbekistan, Tashkent-174, Uzbekistan
}

Received: date / Revised version: date

\begin{abstract}
The neutron-proton mass difference in finite nuclei is studied in the framework of a mediummodified Skyrme model. The possible interplay between the effective nucleon mass in finite nuclei and the Nolen-Schiffer anomaly is discussed. In particular, we find that a correct description of the properties of mirror nuclei leads to a stringent restriction of possible modifications of the nucleon's effective mass in nuclei.
\end{abstract}

PACS. 12.39.Fe Chiral Lagrangians - 21.10.Sf Coulomb energies, analogue states - 21.65.+f Nuclear matter - 14.20.Dh Protons and neutrons

\section{Introduction}

An essential quantity in nuclear physics, the effective neutron-proton mass difference in nuclear matter, $\Delta m_{\mathrm{np}}^{*}$, is still not known empirically [1]. On the other hand, there exist very different theoretical predictions of this quantity for isospin-asymmetric nuclear matter $[2,3,4,5,6,7,8,8$, $9,10,11,12,13,14,15$, where the results differ both qualitatively and quantitatively. Such studies of the effective neutron-proton mass difference inside nuclei may be relevant to resolve the Nolen-Schiffer anomaly (NSA) in nuclear physics 16,17 and for applications in nuclear astrophysics [18,19,20. Although there are many theoretical approaches devoted to the explanation of the NSA [21,32, 22, 23, 24, 25, 26, 27, 28, 29, 30, 31 this phenomenon is still not fully understood.

In this context, we have recently proposed an effective Lagrangian [33] which can be applied to investigate isospin-breaking effects in the baryonic sector of the Skyrme model under medium modifications. In ref. 33 the single nucleon properties and $\Delta m_{\mathrm{np}}^{*}$ are studied in infinite, asymmetric nuclear matter with a spatially constant density, where the surrounding medium influences were taken into account as external parameters. Moreover, the model can be extended to the evaluation of nucleon properties in finite nuclei. Such kind of studies, however with only partial isospin-splitting effects, have already been performed in refs. 34 35. It will be interesting to extend these stud-

1 Thereafter an asterix in an expression indicates that the corresponding quantity is explicitly medium-modified. ies to the strong and electromagnetic isospin-breaking effects in finite nuclei. Consequently, in the present work we consider the neutron-proton mass difference in finite nuclei by studying the single-nucleon effective properties and by trying to explain the Nolen-Schiffer anomaly within a medium-modified Skyrme model.

The paper is organized as follows: In sect. 2 we review the model Lagrangian which was adapted in ref. 33 to skyrmions in asymmetric nuclear matter. Furthermore, we specify the input parameters and the medium functionals, which express the influence of the surrounding environment onto the single-nucleon properties, together with the parameterization of the neutron- and proton-density distributions. Section 3 centers on the spherically symmetric case where a classical skyrmion is located at the center of a spherical nucleus. In particular, we contrast the exact solution of the equations of motion with a variational approximation and compare in sect. 4 the results of both calculations. Next, in sect. 5 we extend our studies to the nonspherical case where the nucleons are located at some finite distance from the center of nucleus. We discuss the consequences of the nonspherical case for the time-dependent Lagrangian, the appropriate classical solutions and the quantization procedure. In sect. 6, we present the pertinent calculations for the electromagnetic part of the neutron-proton mass difference in finite nuclei and in sect. 7 we discuss the results of the nonspherical approximation. Our discussions of the Nolen-Schiffer anomaly are presented in sect. 8 and a summary of the results is given in sect. 9. Finally and for completeness, two 
appendixes are added which elaborate on the nonspherical scenario: the first is devoted to the mass functional and moments of inertia (appendix $\mathrm{A}$ ) and the second to the pertinent charges (appendix B).

\section{Lagrangian of the model}

We start with the Skyrme-model Lagrangian presented in ref. 33

$$
\begin{aligned}
\mathcal{L}^{*}= & \mathcal{L}_{\text {sym }}^{*}+\mathcal{L}_{\mathrm{as}}^{*} \\
\mathcal{L}_{\mathrm{sym}}^{*}= & \mathcal{L}_{2}^{*}+\mathcal{L}_{4}+\mathcal{L}_{\chi \mathrm{SB}}^{*}, \\
\mathcal{L}_{\mathrm{as}}^{*}= & \Delta \mathcal{L}_{\mathrm{mes}}+\Delta \mathcal{L}_{\mathrm{env}}^{*}, \\
\mathcal{L}_{2}^{*}= & \frac{F_{\pi}^{2}}{16}\left\{\left(1+\frac{\chi_{s}^{02}}{m_{\pi}^{2}}\right) \operatorname{Tr}\left(\partial_{0} U \partial_{0} U^{\dagger}\right)\right. \\
& \left.-\left(1-\chi_{p}^{0}\right) \operatorname{Tr}\left(\nabla U \cdot \nabla U^{\dagger}\right)\right\}, \\
\mathcal{L}_{4}= & \frac{1}{32 e^{2}} \operatorname{Tr}\left[U^{\dagger} \partial_{\mu} U, U^{\dagger} \partial_{\nu} U\right]^{2}, \\
\mathcal{L}_{\chi \mathrm{SB}}^{*}= & -\frac{F_{\pi}^{2} m_{\pi}^{2}}{16}\left(1+m_{\pi}^{-2} \chi_{s}^{00}\right) \\
& \times \operatorname{Tr}\left[(U-1)\left(U^{\dagger}-1\right)\right], \\
\Delta \mathcal{L}_{\mathrm{mes}}= & -\frac{F_{\pi}^{2}}{16} \sum_{a=1}^{2} \mathcal{M}_{-}^{2} \operatorname{Tr}\left(\tau_{a} U\right) \operatorname{Tr}\left(\tau_{a} U^{\dagger}\right), \\
\Delta \mathcal{L}_{\mathrm{env}}^{*}= & -\frac{F_{\pi}^{2}}{16} \sum_{a, b=1}^{2} \varepsilon_{a b 3} \frac{\Delta \chi_{s}+\Delta \chi_{p}}{2 m_{\pi}} \\
& \times \operatorname{Tr}\left(\tau_{a} U\right) \operatorname{Tr}\left(\tau_{b} \partial_{0} U^{\dagger}\right),
\end{aligned}
$$

where Einstein's summation convention is always assumed (if not specified otherwise). The chiral $S U(2)$ matrix $U$ has the form $U=\exp \left(2 i \tau_{a} \pi_{a} / F_{\pi}\right)$, where $\pi_{a}(a=1,2,3)$ are the Cartesian isospin-components of the pion field. $F_{\pi}=2 f_{\pi}$ is the pion-decay constant, while $e$ is the dimensionless Skyrme constant. $\mathcal{M}_{-} \equiv \sqrt{\left(m_{\pi^{ \pm}}^{2}-m_{\pi^{0}}^{2}\right) / 2}$ is defined in terms of the masses of the charged and neutral pions, where for the sake of convenience the mass of the neutral pion is denoted as $m_{\pi} \equiv m_{\pi^{0}}$ in (4), (6) and (8). Finally, $\chi_{s}^{00}, \chi_{s}^{02}, \chi_{p}^{0}, \Delta \chi_{s}$, and $\Delta \chi_{p}$ represent the medium functionals which express the influence of the surrounding environment onto the single-skyrmion properties. The numerical values of the input parameters in the present work have slightly been changed in comparison to the work [33. The parameters $F_{\pi}$ and $e$ are fixed in such a way that the free-space (density $\rho=0$ ) PDG-values of the proton and neutron masses $\left(m_{\mathrm{p}}=938.27 \mathrm{MeV}\right.$ and $m_{\mathrm{n}}=939.56 \mathrm{MeV}$ 36 ) together with the empirical (isospin-averaged) mass of the delta $\left(m_{\Delta}=1232 \mathrm{MeV}\right)$ are reproduced2 2 . Furthermore, as input for the free mass of the neutral pion we still use the PDG-value [36]: $m_{\pi^{0}}=$

\footnotetext{
2 Note that in the previous work 33 the input for $F_{\pi}$ and $e$ was determined by a fit to the isospin-averaged masses of both, the nucleon and the $\Delta$.
}

134.977 MeV. All of these choices together induce the following values for the input-parameters: $F_{\pi}=108.783 \mathrm{MeV}$, $e=4.854$, and $m_{\pi^{ \pm}}=135.015 \mathrm{MeV}$; the latter is the strong part of the mass of the charged pion. Note that the dominant electromagnetic contribution to $m_{\pi^{ \pm}}-m_{\pi^{0}}$ is still beyond the scope of the model.

In the Lagrangian (11)-(3) the term $\mathcal{L}_{\text {sym }}^{*}$ expresses the isospin-symmetric part, whereas $\Delta \mathcal{L}_{\text {mes }}$ and $\Delta \mathcal{L}_{\text {env }}^{*}$ are the isospin-breaking terms that arise from the explicit symmetry breaking in the mesonic sector and the isospin asymmetry of the surrounding environment, respectively.

\subsection{Medium functionals}

The medium functionals are given as in 33]:

$$
\begin{aligned}
\chi_{s}^{00} & =\left(\tilde{b}_{0}+\frac{3 k_{\mathrm{F}}}{8 \pi^{2} \eta} \tilde{b}_{0}^{2}\right) \rho, \\
\chi_{s}^{02} & =\left(\tilde{b}_{0}+\frac{3 k_{\mathrm{F}}}{4 \pi^{2} \eta}\left(\tilde{b}_{0}^{2}-\tilde{b}_{1}^{2}\right)\right) \rho, \\
\chi_{p}^{0} & =\frac{2 \pi c^{+}}{1+4 \pi g^{\prime} c^{+}}+\frac{2 \pi c^{-}}{1+4 \pi g^{\prime} c^{-}}, \\
\Delta \chi_{s} & =\tilde{b}_{1} \delta \rho \\
\Delta \chi_{p} & =-\frac{2 \pi m_{\pi}}{\eta m_{\mathrm{N}}} c_{1}\left(\nabla^{2} \delta \rho\right),
\end{aligned}
$$

where

$$
\begin{aligned}
c^{ \pm} & \equiv\left(c_{0} \rho \mp c_{1} \delta \rho\right) \eta^{-1}, \\
\rho(\boldsymbol{r}) & =\rho_{\mathrm{n}}(\boldsymbol{r})+\rho_{\mathrm{p}}(\boldsymbol{r}), \\
\delta \rho(\boldsymbol{r}) & =\rho_{\mathrm{n}}(\boldsymbol{r})-\rho_{\mathrm{p}}(\boldsymbol{r}), \\
\eta & =1+m_{\pi} / m_{\mathrm{N}} .
\end{aligned}
$$

Here $k_{\mathrm{F}}=\left[3 \pi^{2} \rho / 2\right]^{1 / 3}$ is the total Fermi momentum, $\rho_{\mathrm{n}}(\boldsymbol{r})$ and $\rho_{\mathrm{p}}(\boldsymbol{r})$ are the neutron- and proton-distribution densities in the nucleus, $m_{\mathrm{N}}$ is the isospin-averaged nucleon mass: $m_{\mathrm{N}}=\left(m_{\mathrm{p}}+m_{\mathrm{n}}\right) / 2$. The parameters of the medium functionals have the values $\tilde{b}_{0}=-1.206 m_{\pi}^{-1}$, $\tilde{b}_{1}=-1.115 m_{\pi}^{-1}, c_{0}=0.21 m_{\pi}^{-3}, c_{1}=0.165 m_{\pi}^{-3}$ and $g^{\prime}=0.47$ 33]. The relations between the parameters $\tilde{b}_{0,1}$ and the $s$-wave pion nucleon scattering lengths $b_{0,1}$, based on chiral perturbation theory at order $\mathcal{O}\left(m_{\pi}^{3}\right)$, can be found in refs. [37],38. As input for the $p$-wave scattering volumes, $c_{0}$ and $c_{1}$, we use the threshold values of the 'current' SAID analysis $[39]^{3}$.

\subsection{Proton and neutron distributions in finite nuclei}

From now on we will only concentrate on nuclei in the ground state and furthermore consider only nuclei which either are "magic" or which are very near to shell closure.

\footnotetext{
3 For more references and explanations about the chosen values of $\tilde{b}_{0,1}$ and $c_{0,1}$ see ref. 33 .
} 
This allows us to use spherically symmetric approximations to the nucleon distributions inside the nuclei. Consequently, the distribution densities of protons and neutrons in finite nuclei are parameterized in the standard way [40] as

$$
\begin{aligned}
\rho_{\mathrm{p}, \mathrm{n}}(r)= & (Z, A-Z) \frac{3}{4 \pi r_{\mathrm{p}, \mathrm{n}}^{\prime 3}}\left(1+\frac{\pi^{2} a_{\mathrm{p}, \mathrm{n}}^{\prime 2}}{r_{\mathrm{p}, \mathrm{n}}^{\prime 2}}\right)^{-1} \\
& \times \frac{1}{1+\exp \left\{\left(r-r_{\mathrm{p}, \mathrm{n}}^{\prime}\right) / a_{\mathrm{p}, \mathrm{n}}^{\prime}\right\}}
\end{aligned}
$$

in terms of the mass-number- and isospin-dependent parameters

$$
\begin{aligned}
& r_{\mathrm{p}, \mathrm{n}}^{\prime}=r_{\mathrm{p}, \mathrm{n}}^{(0)} A^{1 / 3}+r_{\mathrm{p}, \mathrm{n}}^{(1)}+r_{\mathrm{p}, \mathrm{n}}^{(2)} \lambda, \\
& a_{\mathrm{p}, \mathrm{n}}^{\prime}=a_{\mathrm{p}, \mathrm{n}}^{(1)}+a_{\mathrm{p}, \mathrm{n}}^{(2)} \lambda .
\end{aligned}
$$

In these expressions $A$ is the mass number, $Z$ is the number of protons, and $\lambda=(A-2 Z) / A$ is the isospin-asymmetry parameter. As input for the parameters of the neutronand proton-distributions we take the values

$$
\begin{array}{ll}
r_{\mathrm{p}}^{(0)}=1.2490 \mathrm{fm}, & r_{\mathrm{n}}^{(0)}=1.2131 \mathrm{fm}, \\
r_{\mathrm{p}}^{(1)}=-0.5401 \mathrm{fm}, & r_{\mathrm{n}}^{(1)}=-0.4415 \mathrm{fm}, \\
r_{\mathrm{p}}^{(2)}=-0.9582 \mathrm{fm}, & r_{\mathrm{n}}^{(2)}=0.8931 \mathrm{fm}, \\
a_{\mathrm{p}}^{(1)}=0.4899 \mathrm{fm}, & a_{\mathrm{n}}^{(1)}=0.4686 \mathrm{fm}, \\
a_{\mathrm{p}}^{(2)}=-0.1236 \mathrm{fm}, & a_{\mathrm{n}}^{(2)}=0.0741 \mathrm{fm} .
\end{array}
$$

These values were obtained from calculations based on effective Skyrme interactions used in the description of the properties of finite nuclei [41].

As our main interest is the Nolen-Schiffer anomaly, we will concentrate our investigations to the following pairs of mirror nuclei: ${ }^{15} \mathrm{~N}$ versus ${ }^{15} \mathrm{O},{ }^{17} \mathrm{O}$ versus ${ }^{17} \mathrm{~F},{ }^{39} \mathrm{~K}$ versus ${ }^{39} \mathrm{Ca}$, and finally ${ }^{41} \mathrm{Ca}$ versus ${ }^{41} \mathrm{Sc}$. An important point in the calculation of the effective nucleon properties is the proper definition of the nuclear background density. For example, by adding either a neutron or a proton to the background of the spherical nucleus ${ }^{16} \mathrm{O}$ one can get the nucleus ${ }^{17} \mathrm{O}$ or ${ }^{17} \mathrm{~F}$, respectively. We assume that possible changes in the structure of the ${ }^{16} \mathrm{O}$ core due to the additional valence nucleon are small and can be ignored.

\section{Parameterization of the classical solutions I (spherically symmetric configurations)}

When the skyrmion is located at the center of the nucleus, one can still use the spherically symmetric hedgehog ansatz

$$
U=\exp [i \boldsymbol{\tau} \cdot(\boldsymbol{r} / r) F(r)]
$$

and, following the two-step method of refs. 33, 42, construct the time-dependent Lagrangian in terms of the standard angular velocities $\omega_{i}$ of the collective modes and the constrained angular velocity $a^{*}$. Then the time-dependent
Lagrangian is given as

$$
\begin{aligned}
L^{*} & =\int \mathcal{L}^{*} \mathrm{~d}^{3} r=-M_{\mathrm{NP}}^{*}-\mathcal{M}_{-}^{2} \Lambda_{-}+\frac{\omega^{2}}{2} \Lambda^{*} \\
& +\omega_{3}\left(a^{*} \Lambda^{*}+\Delta^{*}\right)+a^{*}\left(\frac{a^{*}}{2} \Lambda^{*}+\Delta^{*}\right),
\end{aligned}
$$

where

$$
\begin{aligned}
M_{\mathrm{NP}}^{*} & =\pi \int_{0}^{\infty}\left\{\frac{F_{\pi}^{2}}{2}\left(1-\chi_{p}^{0}\right)\left(F_{r}^{2}+\frac{2 S^{2}}{r^{2}}\right)\right. \\
& +\frac{2}{e^{2}}\left(2 F_{r}^{2}+\frac{S^{2}}{r^{2}}\right) \frac{S^{2}}{r^{2}} \\
& \left.+F_{\pi}^{2}\left(m_{\pi}^{2}+\chi_{s}^{00}\right)(1-\cos F)\right\} r^{2} \mathrm{~d} r
\end{aligned}
$$

is the in-medium mass of the solution when it is not perturbed (NP) by any isospin-breaking effects in the mesonic sector or the nuclear environment. The abbreviations $F_{r} \equiv$ $\mathrm{d} F / \mathrm{d} r$ and $S \equiv \sin F$ have been used, where $F=F(r)$ is the chiral profile function of the hedgehog ansatz.

$$
\Lambda_{-}=\frac{2 \pi}{3} F_{\pi}^{2} \int_{0}^{\infty} S^{2} r^{2} \mathrm{~d} r
$$

is a moment-of-inertia-type term resulting from the explicit (strong) isospin-breaking due to the pion masses, whereas

$$
\begin{aligned}
\Lambda^{*} & =\frac{2 \pi}{3} F_{\pi}^{2} \int_{0}^{\infty}\left(1+m_{\pi}^{-2} \chi_{s}^{02}\right) S^{2} r^{2} \mathrm{~d} r \\
& +\frac{8 \pi}{3 e^{2}} \int_{0}^{\infty}\left(F_{r}^{2}+\frac{S^{2}}{r^{2}}\right) S^{2} r^{2} \mathrm{~d} r
\end{aligned}
$$

is the moment of inertia of the skyrmion in the nuclear medium. Finally,

$$
\Delta^{*}=\frac{2 \pi}{3} F_{\pi}^{2} \int_{0}^{\infty} \Delta \alpha S^{2} r^{2} \mathrm{~d} r
$$

with

$$
\Delta \alpha=\frac{1}{2 m_{\pi}}\left(\Delta \chi_{s}+\Delta \chi_{p}\right)
$$

is the isospin-breaking factor due to the isospin asymmetry of the nuclear environment. We note that on top of the terms considered in the previous refs. 33,42 also density gradients of the surrounding environment are relevant which result from the $p$-wave pion-nucleus scattering.

In order to recover the minimization functional in the classical approximation $\left(\omega_{i} \equiv 0\right)$, one applies the constraint 42

$$
a^{* 2}=2 \mathcal{M}_{-}^{2} \Lambda_{-} / \Lambda^{*}
$$


such that from eq. (21) the Lagrangian

$$
L^{*}=-M_{\mathrm{NP}}^{*}+a^{*} \Delta^{*}
$$

is generated for vanishing $\omega_{i}$. The variation of the Lagrangian (28) gives the pertinent equation of motion for the hedgehog profile function $F(r)$,

$$
\begin{aligned}
& F_{\pi}^{2}\left(1-\chi_{p}^{0}\right)\left(r^{2} F_{r r}+2 r F_{r}-S_{2}\right)-F_{\pi}^{2} \chi_{p, r}^{0} r^{2} F_{r} \\
& +\frac{4}{e^{2}}\left[2 S^{2} F_{r r}+S_{2}\left(F_{r}^{2}-\frac{S^{2}}{r^{2}}\right)\right] \\
& -F_{\pi}^{2}\left(m_{\pi}^{2}+\chi_{s}^{00}\right) S r^{2}+\frac{2 a^{*} F_{\pi}^{2}}{3} \Delta \alpha S_{2} r^{2}=0,
\end{aligned}
$$

where the additional abbreviations $F_{r r}=\mathrm{d}^{2} F / \mathrm{d} r^{2}, S_{2}=$ $\sin 2 F$ and $\chi_{p, r}^{0}=\mathrm{d} \chi_{p}^{0} / \mathrm{d} r$ were introduced. The boundary conditions

$$
\begin{aligned}
& \lim _{r \rightarrow 0} F(r)=\pi-C r, \\
& \lim _{r \rightarrow \infty} F(r)=D\left(1+m_{\pi} r\right) \frac{e^{-m_{\pi} r}}{r^{2}},
\end{aligned}
$$

where $C$ and $D$ are constants, correspond to a classical soliton of baryon number $B=1$.

As an alternative, we will also use the following trial function for approximating the exact solutions of the abovegiven differential equation (29):

$$
\begin{aligned}
& F(r)=2 \arctan \left\{\frac{r_{0}^{2}}{r^{2}}\left(1+m_{\pi} r\right)\right\} e^{-f(r) r}, \\
& f(r)=\beta_{0}+\beta_{1} e^{\beta_{2} r^{2}} .
\end{aligned}
$$

Here $r_{0}, \beta_{0}, \beta_{1}$, and $\beta_{2}$ are variational parameters. Note that our trial function $F(r)$ differs from the widely used trial function $2 \arctan \left\{r_{0}^{2} / r^{2}\right\}$, since it takes into account not only the asymptotical behavior at the origin and the far-distance region, but approximates also the behavior in the intermediate range. The trial ansatz will be useful in our following studies of approximate solutions in the nonspherical case.

As in the previous work 33 the final value of $a^{*}$ is found by an iteration procedure, starting at $a^{*}=0$.

Whenever the skyrmion can be parameterized as a spherically symmetric hedgehog configuration, the quantization procedure and the expressions for the electromagnetic part of the neutron-proton mass difference remain the same as given in in ref. 33], except for the fact that some global medium functionals acquire a local density dependence (see eq.s (91)-(16)) and that the expression of $\Delta^{*}$ also has an additional contribution from $p$-wave pionnucleus scattering 4 .

\section{Results in the spherically symmetric approximation}

The results arising from (i) the direct solutions of equations of motion (29) and (ii) from the approximate variational procedure with the trial function (31) are presented

\footnotetext{
${ }^{4}$ Compare the present expressions (25) and (26) with the expressions (40) and (38) of ref. 33.
}

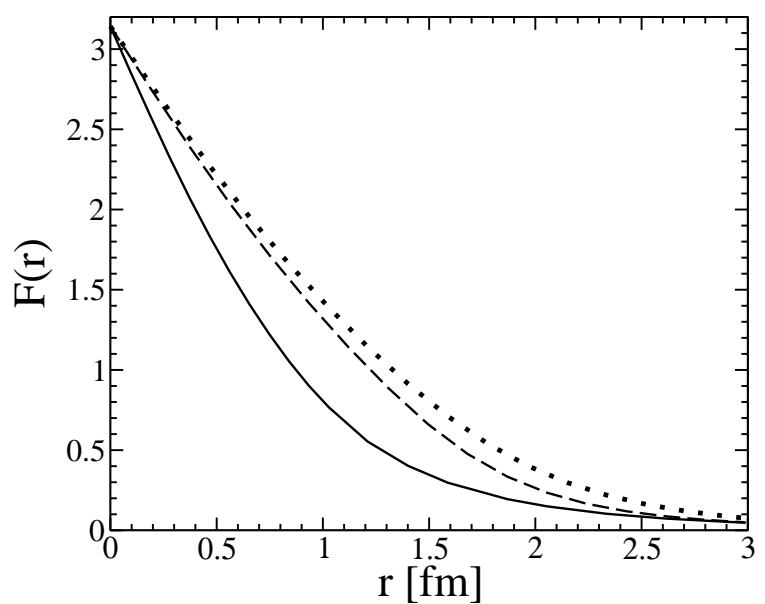

Fig. 1. The solutions of eq. (29). The solid curve represents the profile function of a skyrmion in free space, the dashed curve stands for the profile function of a skyrmion located at the center of an ${ }^{16} \mathrm{O}$ background, and the dotted curve represents the case of a skyrmion located at the center of a ${ }^{40} \mathrm{Ca}$ core. The approximate trial function (31) gives almost identical results (indistinguishable at the scale of the figure) for each of these three cases.

in Table 1 for several nuclei. One can see that the trial function (31) very well approximates the actual solutions of the model. The deviations from the exactly calculated static properties of the nucleon due to the approximation (31) are in the range of $0.01 \div 5.4 \%$. This can be seen by comparing the lines (i) and (ii) for each nucleus presented in Table[1. In free space the deviations are even smaller: the maximum deviation is in $\Delta m_{\mathrm{np}}$ and is approximately equal to $2 \%$.

As expected, in accordance to our previous calculations for infinite nuclear matter in the isospin symmetric case [33, the neutron-proton mass difference is slightly increased in comparison to the free space case. Also note that in this model the effective nucleon mass has a strongly decreased value at the center of nucleus. We will return to this point later on.

For illustrative purposes the solutions of the differential equation (29) for a skyrmion in free space as well as inside the nuclei ${ }^{16} \mathrm{O}$ and ${ }^{40} \mathrm{Ca}$ are shown in fig. 1. Note that the profile function is rather insensitive to small density variations: the solutions of the nuclei ${ }^{14} \mathrm{~N}$ and ${ }^{16} \mathrm{O}$ or ${ }^{38} \mathrm{~K}$ and ${ }^{40} \mathrm{Ca}$, respectively, almost coincide. Finally, from the results presented in Table 1, it is clear that the trial function (31) with appropriately chosen parameters is almost as good as the true solution. For this reason the trial functions are not presented in fig. 1, since graphically they cannot be distinguished from their exact counterparts.

\section{Nonspherical solutions}

\subsection{Time-dependent Lagrangian}

When the skyrmion is located at some finite distance from the center of the nucleus, the spherically symmetric hedge- 
Table 1. The values of the variational parameters and the static properties of nucleons when the pertinent skyrmion is either in free space or added to the center of a finite (background) nucleus. Here $m_{\mathrm{p}}^{*}$ is the in-medium proton mass, $\Delta m_{\mathrm{np}}^{*}$ is the in-medium neutron-proton mass difference and $\Delta m_{\mathrm{np}}^{*(\mathrm{EM})}$ is its electromagnetic part; $\mu_{\mathrm{p}}^{*}$ and $\mu_{\mathrm{n}}^{*}$ are the in-medium proton and neutron magnetic moments in units of nucleon Bohr magnetons (n.m.) in free space; $\left\langle r^{2}\right\rangle_{\mathrm{E}, \mathrm{S}}^{* 1 / 2}$ and $\left\langle r^{2}\right\rangle_{\mathrm{E}, \mathrm{V}}^{* 1 / 2}$ are the in-medium isoscalar $(\mathrm{S})$ and isovector $(\mathrm{V})$ charge radii of the nucleons. For a given nucleus we present (i) the results corresponding to the exact solution of the differential equation (29) and (ii) the results of the approximate variational procedure where the trial function (31) has been used.

\begin{tabular}{|c|c|c|c|c|c|c|c|c|c|c|c|c|}
\hline \multicolumn{2}{|c|}{ Element } & \multirow{2}{*}{$\begin{array}{r}\begin{array}{c}r_{0} \\
{[\mathrm{fm}]}\end{array} \\
\end{array}$} & \multirow{2}{*}{$\begin{array}{r}10 \beta_{0} \\
{\left[m_{\pi}\right]}\end{array}$} & \multirow{2}{*}{$\begin{array}{c}\beta_{1} \\
{\left[m_{\pi}\right]}\end{array}$} & $\begin{array}{c}\beta_{2} \\
{\left[m_{\pi}^{2}\right]}\end{array}$ & \multirow{2}{*}{$\begin{array}{c}m_{\mathrm{p}}^{*} \\
{[\mathrm{MeV}]} \\
938.268\end{array}$} & \multirow{2}{*}{$\begin{array}{c}\Delta m_{\mathrm{np}}^{*} \\
{[\mathrm{MeV}]} \\
1.291\end{array}$} & \multirow{2}{*}{$\begin{array}{l}m_{\mathrm{np}}^{*(\mathrm{EM})} \\
{[\mathrm{MeV}]} \\
-0.686\end{array}$} & \multirow{2}{*}{$\begin{array}{c}\begin{array}{c}\mu_{\mathrm{p}}^{*} \\
{[\mathrm{n} . \mathrm{m} .]}\end{array} \\
1.963\end{array}$} & \multirow{2}{*}{$\begin{array}{c}\begin{array}{c}\mu_{\mathrm{n}}^{*} \\
{[\mathrm{n} . \mathrm{m} .]}\end{array} \\
-1.236\end{array}$} & \multirow{2}{*}{$\begin{array}{l}\left\langle r^{2}\right\rangle_{\mathrm{E}, \mathrm{S}}^{* 1 / 2} \\
{[\mathrm{fm}]} \\
0.481\end{array}$} & \multirow{2}{*}{$\begin{array}{l}\left\langle r^{2}\right\rangle_{\mathrm{E}, \mathrm{V}}^{* 1 / 2} \\
{[\mathrm{fm}]}\end{array}$} \\
\hline free & (i) & & & & - & & & & & & & \\
\hline space & (ii) & 0.954 & 0.075 & 1.311 & -0.009 & 938.809 & 1.313 & -0.687 & 1.966 & -1.241 & 0.481 & 0.739 \\
\hline \multirow[b]{2}{*}{${ }^{14} \mathrm{~N}$} & (i) & - & - & - & - & 593.285 & 1.668 & -0.526 & 2.355 & -1.276 & 0.656 & 0.850 \\
\hline & (ii) & 1.393 & 0.076 & 0.920 & 0.226 & 598.505 & 1.655 & -0.536 & 2.230 & -1.209 & 0.648 & 0.810 \\
\hline${ }^{16} \mathrm{O}$ & (ii) & 1.426 & 0.076 & 0.907 & 0.219 & 590.175 & 1.685 & -0.527 & 2.341 & -1.232 & 0.660 & 0.825 \\
\hline \multirow[b]{2}{*}{${ }^{38} \mathrm{~K}$} & (i) & - & - & - & - & 558.088 & 1.804 & -0.480 & 2.584 & -1.422 & 0.722 & 0.942 \\
\hline & (ii) & 1.493 & 0.076 & 0.841 & 0.153 & 559.957 & 1.802 & -0.485 & 2.550 & -1.377 & 0.718 & 0.910 \\
\hline \multirow[b]{2}{*}{${ }^{40} \mathrm{Ca}$} & (i) & - & - & - & - & 557.621 & 1.804 & -0.478 & 2.569 & -1.428 & 0.724 & 0.947 \\
\hline & (ii) & 1.489 & 0.076 & 0.839 & 0.149 & 559.378 & 1.802 & -0.483 & 2.557 & -1.383 & 0.720 & 0.914 \\
\hline
\end{tabular}

hog ansatz cannot be used anymore, since the background profile is not spherically symmetric. Thus there exist further deformations in the isotopic as well as in the coordinate space [34,35]. In this case one should use a more generalized ansatz for the matrix $U$ :

$$
U(\boldsymbol{r}-\boldsymbol{R})=\exp [i \boldsymbol{\tau} \cdot \boldsymbol{N}(\boldsymbol{r}-\boldsymbol{R}) P(\boldsymbol{r}-\boldsymbol{R})],
$$

where $\boldsymbol{R}$ is the distance between the geometrical centers of the skyrmion and the nucleus (see fig. 2). Furthermore note that the argument of the profile function $P$ depends on the direction in coordinate space 5 . For consistency, the isotopic vector $\boldsymbol{N}$ must also be generalized as

$$
\boldsymbol{N}(\boldsymbol{r}-\boldsymbol{R})=\left(\begin{array}{l}
\sin \Theta(\boldsymbol{r}-\boldsymbol{R}) \cos \varphi \\
\sin \Theta(\boldsymbol{r}-\boldsymbol{R}) \sin \varphi \\
\cos \Theta(\boldsymbol{r}-\boldsymbol{R})
\end{array}\right)
$$

in terms of a distance- and direction-dependent generalized angle $\Theta=\Theta(\boldsymbol{r}-\boldsymbol{R})$. Furthermore, due to the azimuthal symmetry of the problem (again see fig. 2), one can restrict the form of both functions as

$$
P=P(|\boldsymbol{r}-\boldsymbol{R}|, \theta), \quad \Theta=\Theta(|\boldsymbol{r}-\boldsymbol{R}|, \theta)
$$

and put the origin of the coordinate system at the geometrical center of the skyrmion: $\boldsymbol{r}^{\prime}=\boldsymbol{r}-\boldsymbol{R}$. For notational convenience we rename $\boldsymbol{r}^{\prime}$ to $\boldsymbol{r}$, keeping in mind that the medium functionals acquire an $\boldsymbol{R}$ dependence (see appendix $\mathrm{A}$.

\footnotetext{
${ }^{5}$ Our notation distinguishes between spherically symmetric profile functions $(F)$ and direction-dependent ones $(P)$.

6 This redefinition procedure is explained in more detail in ref. 34.
}

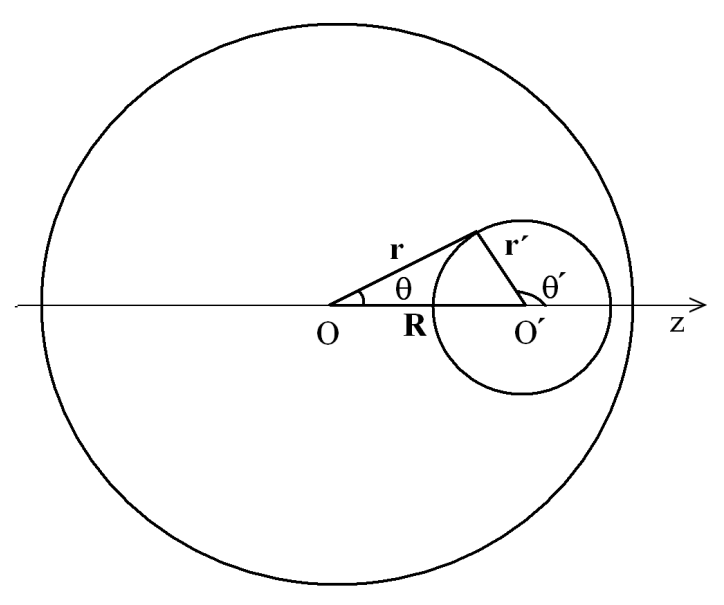

Fig. 2. A sketch of a skyrmion located inside a finite nucleus. Here $\boldsymbol{R}$ is the distance between the center of the nucleus (O) and the geometrical center of the skyrmion $\left(\mathrm{O}^{\prime}\right)$; the vectors (angles) $\boldsymbol{r}(\theta)$ and $\boldsymbol{r}^{\prime}\left(\theta^{\prime}\right)$ refer to the body-fixed coordinates of the nucleus and skyrmion, respectively. Without loss of generality both coordinate systems can be orientated in such a way that their $z$-axes coincide.

In order to apply the collective-coordinate-method to the configuration given in (32)-(34) we consider the following independent rotations in coordinate space and in isotopic space

$$
P=P\left(\mathcal{R}^{-1}(t) \boldsymbol{r}\right), \quad \boldsymbol{N}=\mathcal{I}(t) \boldsymbol{N}\left(\mathcal{R}^{-1}(t) \boldsymbol{r}\right)
$$


where $\mathcal{R}$ and $\mathcal{I}$ are rotational and iso-rotational matrices, respectively. Applying these time-dependent rotations to the two-step method of ref. 33, one arrives at a Lagrangian which - after a spatial integration - is given as

$$
\begin{aligned}
L^{*} & =-M_{\mathrm{NP}}^{*}-\mathcal{M}_{-}^{2} \Lambda_{\mathrm{mes}}+\frac{\omega_{1}^{2}+\omega_{2}^{2}}{2} \Lambda_{\omega \omega, 12}^{*} \\
& -\left(\omega_{1} \Omega_{1}+\omega_{2} \Omega_{2}\right) \Lambda_{\omega \Omega, 12}^{*}+\frac{\Omega_{1}^{2}+\Omega_{2}^{2}}{2} \Lambda_{\Omega \Omega, 12}^{*} \\
& +\frac{\left(\omega_{3}-\Omega_{3}+a^{*}\right)^{2}}{2} \Lambda_{\omega \Omega, 33}^{*} \\
& +\left(\omega_{3}-\Omega_{3}+a^{*}\right) \Lambda_{\mathrm{env}}^{*} .
\end{aligned}
$$

Here $\omega_{i}$ and $\Omega_{i}$ are the angular velocities in isotopic and coordinate space, respectively. The explicit expressions for the functionals, $M_{\mathrm{NP}}^{*}[P, \Theta]$ and each $\Lambda[P, \Theta]$, can be found in appendix $\mathrm{A}$

\subsection{Parameterization of the classical solutions II}

As in our previous works 33,42 , by incorporating the constraint for the angular-velocity parameter $a^{*}$, which in the azimuthally symmetric case has the form

$$
a^{* 2}=2 \mathcal{M}_{-}^{2} \frac{\Lambda_{\mathrm{mes}}}{\Lambda_{\omega \Omega, 33}^{*}}
$$

one arrives at the following Lagrange functional at the classical level:

$$
L^{*}=-M_{\mathrm{NP}}^{*}+a^{*} \Lambda_{\mathrm{env}}^{*}
$$

In order to extremize the Lagrange functional (38), one would have to solve the complicated system of coupled partial differential equations that arises from the variation of the Lagrangian. To avoid all the unnecessary technical difficulties connected with a numerical solution of that equation system, we rather use an approximate variational procedure that is specified as follows: first of all, as fig. 1] indicates, the profile function is only weakly dependent on the density of the medium (compare the solid and dashed curves of fig. 1) and almost insensitive to small density changes (compare the dashed and dotted curves of fig. (1). Consequently, we can apply the approximations

$$
\begin{aligned}
& P(r, \theta)=2 \arctan \left\{\frac{r_{0}^{2}}{r^{2}}\left(1+m_{\pi} r\right)(1+u(\theta))\right\} e^{-f(r) r}, \\
& \Theta(r, \theta)=\theta+\zeta(r, \theta),
\end{aligned}
$$

where the special case $P(r, 0)=F(r)$ is the spherically symmetric profile function (in the form as presented in eq. (310) and where the functions $u$ and $\zeta$ satisfy the inequalities $|u|<1$ and $|\zeta|<1$ in the regions $r \in[0, \infty)$ and $\theta \in[0, \pi]$. Following the ideas of ref. [34] we use for the function $u$ the following ansatz

$$
u(\theta)=\sum_{n=1}^{\infty} \gamma_{n} \cos ^{n} \theta
$$

where the $\left\{\gamma_{n}\right\}$ are variational parameters and the cosine functions are chosen to maintain periodicity in $\theta$. Similarly, $\zeta$ can be chosen as

$$
\zeta(r, \theta)=r e^{-\delta_{0}^{2} r^{2}} \sum_{n=1}^{\infty} \delta_{n} \sin 2 n \theta,
$$

where the $\left\{\delta_{n}\right\}$ are also variational parameters. Note that the arguments of the sine-functions are chosen to be a multiple of $2 \theta$ in order to avoid singularities of the form $\sin \Theta / \sin \theta$ in the Lagrange functional (38) (see appendix A for the explicit form of the Lagrange functional). Furthermore, the $r$ dependence of $\zeta$ is chosen in such a way that the equalities $\Theta(0, \theta)=\theta$ and $\Theta(\infty, \theta)=\theta$ are reproduced.

Finally, in terms of the trial functions (39), (40) and (41), the Lagrange functional (38) will be extremized.

\subsection{Quantization of the nonspherical solutions}

Defining canonical conjugate variables in the body-fixed reference system

$$
T_{i}=\frac{\partial L^{*}}{\partial \omega_{i}} \quad \text { and } \quad J_{i}=\frac{\partial L^{*}}{\partial \Omega_{i}},
$$

one obtains from the time-dependent Lagrangian (36) the Hamiltonian:

$$
\begin{aligned}
\hat{H} & =M_{\mathrm{NP}}^{*}+\mathcal{M}_{-}^{2} \Lambda_{\mathrm{mes}}+\frac{\Lambda_{\mathrm{env}}^{* 2}}{2 \Lambda_{\omega \Omega, 33}^{*}} \\
& +\frac{\left(\hat{T}_{1}^{2}+\hat{T}_{2}^{2}\right) \Lambda_{\Omega \Omega, 12}^{*}+\left(\hat{J}_{1}^{2}+\hat{J}_{2}^{2}\right) \Lambda_{\omega \omega, 12}^{*}}{2\left(\Lambda_{\omega \omega, 12}^{*} \Lambda_{\Omega \Omega, 12}^{*}-\Lambda_{\omega \Omega, 12}^{* 2}\right)} \\
& +\frac{\left(\hat{T}_{1} \hat{J}_{1}+\hat{T}_{2} \hat{J}_{2}\right) \Lambda_{\omega \Omega, 12}^{*}}{\Lambda_{\omega \omega, 12}^{*} \Lambda_{\Omega \Omega, 12}^{*}-\Lambda_{\omega \Omega, 12}^{* 2}} \\
& +\frac{\hat{T}_{3}^{2}}{2 \Lambda_{\omega \Omega, 33}^{*}}-\left(a^{*}+\frac{\Lambda_{\mathrm{env}}^{*}}{\Lambda_{\omega \Omega, 33}^{*}}\right) \hat{T}_{3} .
\end{aligned}
$$

By sandwiching the Hamiltonian between the appropriate baryon states $\left|T, T_{3} ; J, J_{3}=-T_{3}\right\rangle$ one determines the energy of a nucleon inside a nucleus as

$$
\begin{aligned}
E & =M_{\mathrm{NP}}^{*}+\mathcal{M}_{-}^{2} \Lambda_{\mathrm{mes}}+\frac{\Lambda_{\mathrm{env}}^{* 2}}{2 \Lambda_{\omega \Omega, 33}^{*}} \\
& +\frac{\Lambda_{\Omega \Omega, 12}^{*}+\Lambda_{\omega \omega, 12}^{*}-2 \Lambda_{\omega \Omega, 12}^{*}}{2\left(\Lambda_{\omega \omega, 12}^{*} \Lambda_{\Omega \Omega, 12}^{*}-\Lambda_{\omega \Omega, 12}^{* 2}\right)}\left(T(T+1)-T_{3}^{2}\right) \\
& +\frac{T_{3}^{2}}{2 \Lambda_{\omega \Omega, 33}^{*}}-\left(a^{*}+\frac{\Lambda_{\mathrm{env}}^{*}}{\Lambda_{\omega \Omega, 33}^{*}}\right) T_{3} .
\end{aligned}
$$

Consequently, the strong part of the neutron-proton mass difference in the interior of a nucleus takes the form

$$
\Delta m_{\mathrm{np}}^{*(\text { strong })}=a^{*}+\frac{\Lambda_{\mathrm{env}}^{*}}{\Lambda_{\omega \Omega, 33}^{*}} .
$$




\section{Electromagnetic part of the neutron-proton mass difference in nonspherically deformed states}

The electric (E) and magnetic (M) form factors of the nucleon are defined through the expressions

$$
\begin{aligned}
G_{\mathrm{E}}^{*}\left(\boldsymbol{q}^{2}\right) & =\frac{1}{2} \int \mathrm{d}^{3} r e^{i \boldsymbol{q} \cdot \boldsymbol{r}} j^{0}(\boldsymbol{r}), \\
G_{\mathrm{M}}^{*}\left(\boldsymbol{q}^{2}\right) & =\frac{m_{\mathrm{N}}}{2} \int \mathrm{d}^{3} r e^{i \boldsymbol{q} \cdot \boldsymbol{r}}[\boldsymbol{r} \times \boldsymbol{j}(\boldsymbol{r})],
\end{aligned}
$$

where $\boldsymbol{q}^{2}$ is the squared momentum transfer. Furthermore, $j^{0}$ and $\boldsymbol{j}$ correspond to the time and space components of the properly normalized sum of the baryonic current $B_{\mu}^{*}$ and the third component of the isovector current $\boldsymbol{V}_{\mu}^{*}$ of the Skyrme model.

For the problem at hand, it is advantageous to expand the plane wave factor in (46), 7 as

$$
e^{i \boldsymbol{q} \cdot \boldsymbol{r}}=e^{i q r \cos \theta}=\sum_{l=0}^{\infty} i^{l}(2 l+1) P_{l}(\cos \theta) J_{l}(q r),
$$

in terms of Legendre polynomials $P_{l}$ and spherical Bessel functions $J_{l}$. In this way we get the final expressions for the electromagnetic form factors

$$
G_{a}^{* b}\left(q^{2}\right)=\sum_{l=0}^{\infty} i^{l}(2 l+1) G_{a, l}^{* b}\left(q^{2}\right) .
$$

Here

$$
G_{a, l}^{* b}\left(q^{2}\right)=\int \mathrm{d}^{3} r \rho_{a}^{b}(r, \theta) P_{l}(\cos \theta) J_{l}(q r)
$$

are partial form factors, where the label $a$ stands either for isoscalar $(\mathrm{S})$ or isovector $(\mathrm{V})$ form factors, and the label $b$ denotes either electric (E) or magnetic (M) form factors. Explicit expressions of the corresponding charge $\left(\rho_{\mathrm{E}}\right)$ and magnetic $\left(\rho_{\mathrm{M}}\right)$ densities are given in appendix B As actual calculations show, the absolute values of the partial form factors decrease quickly with increasing partial wave number $l$ within the present approach.

Finally, applying the formula 43 .

$$
\begin{aligned}
\Delta m_{\mathrm{np}}^{*(\mathrm{EM})}= & -\frac{4 \alpha}{\pi} \int_{0}^{\infty} \mathrm{d} q\left\{G_{\mathrm{E}}^{\mathrm{S} *}\left(\boldsymbol{q}^{2}\right) G_{\mathrm{E}}^{\mathrm{V} *}\left(\boldsymbol{q}^{2}\right)\right. \\
& \left.-\frac{\boldsymbol{q}^{2}}{2 m_{\mathrm{N}}^{2}} G_{\mathrm{M}}^{\mathrm{S} *}\left(\boldsymbol{q}^{2}\right) G_{\mathrm{M}}^{\mathrm{V} *}\left(\boldsymbol{q}^{2}\right)\right\}
\end{aligned}
$$

where $\alpha \approx 1 / 137$ is the electromagnetic fine structure constant, one can calculate the medium-dependent electromagnetic part of the neutron-proton mass difference.

\footnotetext{
7 We can rotate the total system, background nucleus and skyrmion, always such that the $z$-axis of the reference frame coincides with the $z$-direction of the coordinate system of the skyrmion in the body-fixed frame.
}

\section{Results in the nonspherical approximation}

First of all let us remark that numerically the transitions

$$
\lim _{R \rightarrow 0} \Theta(r, \theta) \rightarrow \theta \quad \text { and } \quad \lim _{R \rightarrow 0} P(r, \theta) \rightarrow F(r)
$$

are smooth when the nucleon 'moves' to the center of the nucleus, and that in this case the exact solution is reproduced with high accuracy, see Table 1. One might therefore expect that the trial functions (39)-(41) are not too far from the the true solutions even when the distance between the center of the skyrmion and the center of the nucleus is not equal to zero, $R \neq 0$.

Obviously, the variational parameters $r_{0},\left\{\beta_{n}\right\},\left\{\gamma_{n}\right\}$ and $\left\{\delta_{n}\right\}$ depend on the distance $R$ between the centers of the skyrmion and the nucleus. Note that the strength of the parameter $\gamma_{n}$ decreases with increasing index $n$. For example, $\gamma_{3}$ is one order magnitude smaller than $\gamma_{1}$ at those distances where the deviations of the skyrmion from the spherical form is maximal. The strength of $\delta_{n}$ decreases even more rapidly with increasing $n$ : $\delta_{2}$ is one order of magnitude smaller than $\delta_{1}$ at the above-mentioned distances and $\delta_{3}$ is almost zero for all values of $R$. For performing high accuracy calculations it is therefore sufficient to retain the $\gamma_{n}$ parameters up-to-and-including $\gamma_{4}$ and the $\delta_{n}$ parameters up-to-and-including $\delta_{2}$.

The behavior of the static properties of the nucleons in nuclei and the corresponding variational parameters are discussed in a more detail in ref. 34. Qualitatively, we have similar results in the present approach. For example, the in-medium mass of the proton starts with the effective value presented in Table 1 when it is near the center of the nucleus. Then it increases monotonically with increasing distance between its center and the center of the nucleus until it approaches the free space value at the border of the nucleus, see fig. 3 ,

We will come back to the discussion of the effective nucleon mass in sect.8. Here, however, we concentrate on the effective in-medium neutron-proton mass difference. The behavior of $\Delta m_{\mathrm{np}}^{* \text { (strong) }}$ inside several nuclei is presented in fig. 4. The strong part of the effective mass difference has a non-monotonic behavior. This is a consequence of the density now being a local quantity and of the appearance of additional isospin-breaking contributions due to the density gradients arising from the $p$-wave pion-nucleus scattering (see eq. (26) ). One can explicitly see in fig. (4 that at the surface of the nucleus, where the density gradients are large and the local isospin asymmetry is high, the value of $\Delta m_{\mathrm{np}}^{* \text { (strong) }}$ is at an extremum.

In fig. [5the electromagnetic part of the neutron-proton mass difference is shown for several nuclei. The variations in the electromagnetic part of the neutron-proton mass differences are small compared with their strong counterparts. Qualitatively, their behavior is the same for all nuclei as in the case of strong part. Quantitatively, both parts, $\Delta m_{\mathrm{np}}^{* \text { (strong) }}$ and $\Delta m_{\mathrm{np}}^{* \text { (EM) }}$, are mainly governed by the (local) behavior of the total nuclear density and therefore differ if the total densities of the investigated nuclei are different. 


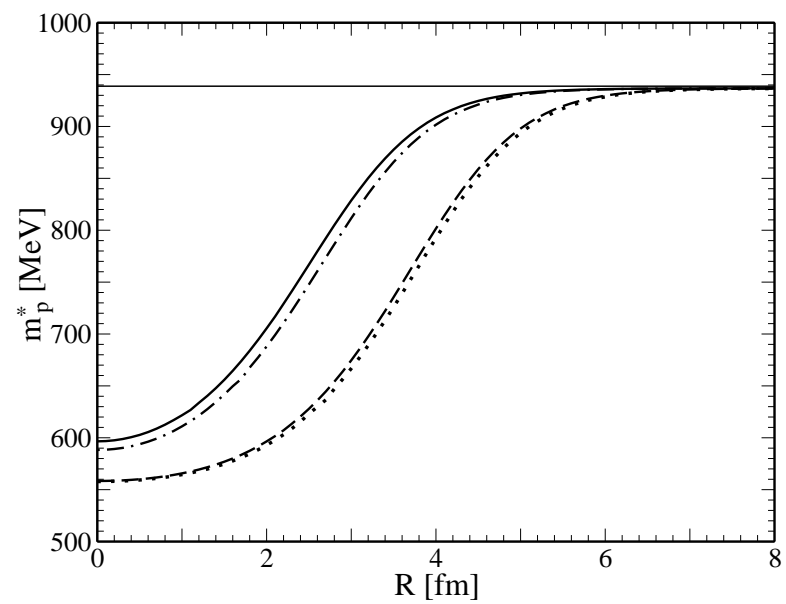

Fig. 3. The dependence of the effective mass of the proton $m_{\mathrm{p}}^{*}$ on the distance $R$ between the centers of the skyrmion and the nucleus. The solid curve represents the result for an additional skyrmion embedded into a ${ }^{14} \mathrm{~N}$ core, the dot-dashed curve stands for the case of ${ }^{16} \mathrm{O}$, the dashed curve refers to the case of ${ }^{38} \mathrm{~K}$ and dotted curve refers to the case of ${ }^{40} \mathrm{Ca}$. The horizontal line indicates the pertinent free space value of the proton mass, see line (ii) in Table 1 i.e. $m_{\mathrm{p}}=938.81 \mathrm{MeV}$.

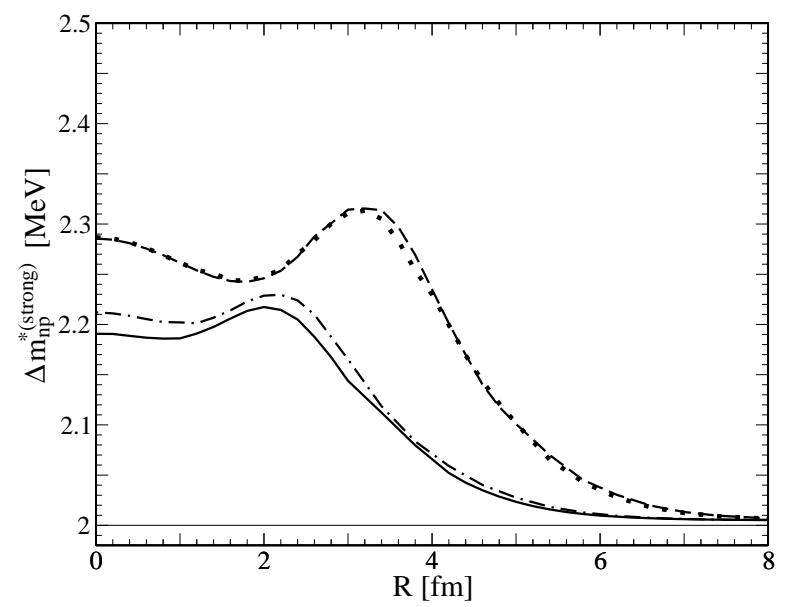

Fig. 4. The dependence of $\Delta m_{\mathrm{np}}^{* \text { (strong) }}$ on the distance $R$ between the centers of the skyrmion and the nucleus. The notations are same as in fig. 3 The horizontal line indicates the free space value of $\Delta m_{\mathrm{np}}^{\text {(strong) }}=2 \mathrm{MeV}$ (see the corresponding line (ii) in Table 1).

Finally, in fig. 6] the dependence of the total neutronproton mass difference $\Delta m_{\mathrm{np}}^{*}$ on the distance $R$ is shown for several nuclei.

\section{Calculation of the Nolen-Schiffer anomaly}

A long standing problem in nuclear physics is the NolenSchiffer anomaly (NSA) observed in mirror nuclei [16, 17]. Let us consider this point in more detail and show how the NSA can be treated in the present framework.

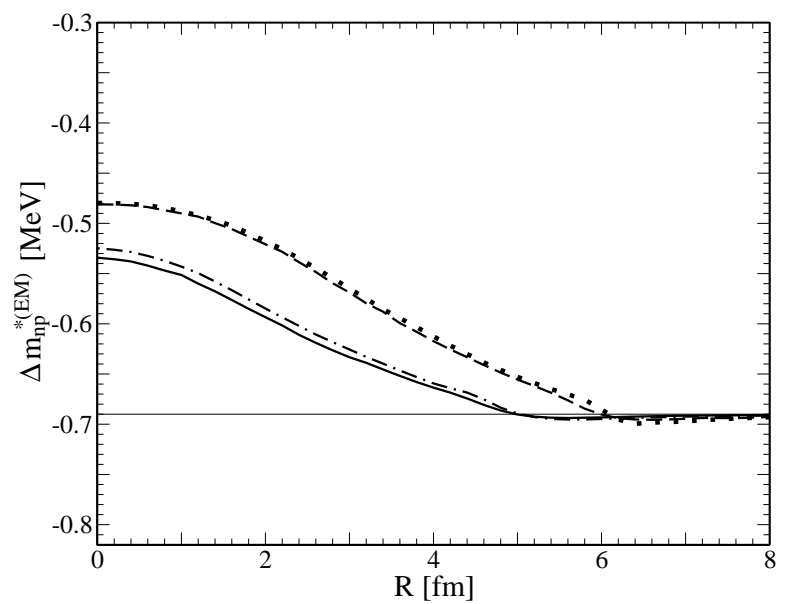

Fig. 5. The dependence of $\Delta m_{\mathrm{np}}^{*(\mathrm{EM})}$ on the distance $R$ between the centers of the skyrmion and the nucleus. The notations are same as in fig. 3. The horizontal line indicates the free space value of $\Delta m_{\mathrm{np}}^{(\mathrm{EM})}=-0.69 \mathrm{MeV}$ (see the corresponding line (ii) in Table 1).

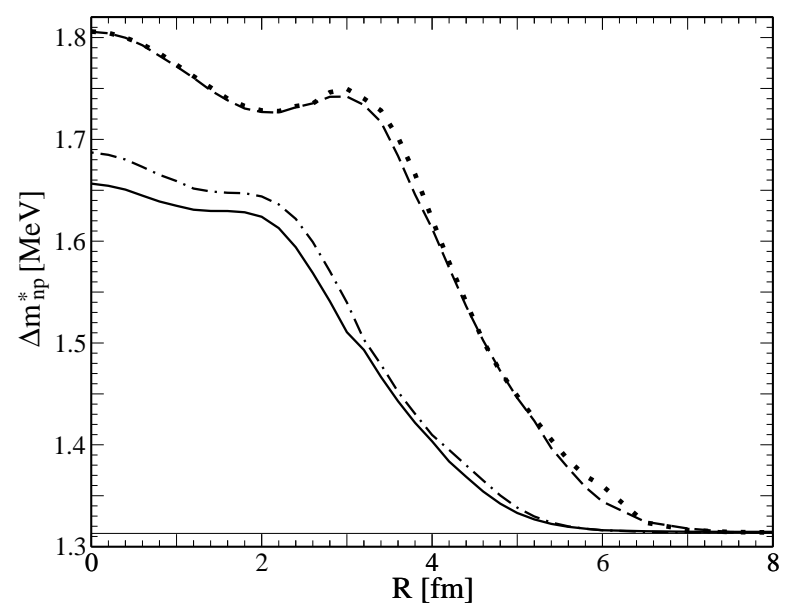

Fig. 6. The total neutron-proton mass difference $\Delta m_{\mathrm{np}}^{*}$ in nuclei. The notations are same as in fig. 3 The horizontal line indicates the free space value of $\Delta m_{\mathrm{np}}=1.31 \mathrm{MeV}$ (see the corresponding line (ii) in Table 1).

The mass difference between mirror nuclei which differ by one unit in their charges, $Z_{1}-Z_{2}=1$, is usually split into two contributions

$$
\Delta M \equiv{ }_{Z+1}^{A} \mathbf{M}_{N}-{ }_{Z}^{A} \mathbf{M}_{N+1}=\Delta E_{\mathrm{EM}}-\Delta m_{\mathrm{np}}^{*},
$$

where $\Delta E_{\mathrm{EM}}$ is the computed Coulomb energy difference, including various corrections (by, e.g., the exchange term, the center-of-mass motion, finite size effects of the proton and neutron charges, magnetic interactions, vacuum effects, the dynamical effect of the neutron-proton mass difference, and short-range two-body correlations). Furthermore, the quantity $\Delta m_{\mathrm{np}}^{*}$ is the effective neutron-proton mass difference. The measurement of the mass difference $\Delta M$ and the theoretical calculation of $\Delta E_{\mathrm{EM}}$ can be done with great accuracy (within $1 \%$ error) [17. Furthermore 
if one assumes that $\Delta m_{\mathrm{np}}^{*}$ is constant and equal to its free space value, then eq. (51) will not be satisfied. This phenomenon is called the Nolen-Schiffer anomaly, persistent throughout the periodic table. Quantitatively, the NSA ranges between few hundred keV for the lightest nuclei up to one $\mathrm{MeV}$ or more for the heaviest nuclei. A possible resolution of the anomaly is the assumption that the effective neutron-proton mass difference $\Delta m_{\mathrm{np}}^{*}$ would decrease with increasing mass number $A$. Consequently, the NSA would be $\Delta_{\mathrm{NSA}}=\Delta m_{\mathrm{np}}-\Delta m_{\mathrm{np}}^{*}$.

Within the present approach $\Delta m_{\mathrm{np}}^{*}$ has a local $R$ dependence according to the location of the nucleons inside the nuclei as represented in fig. 6. In order to compare our results with the experimental data one has to average the value of $\Delta m_{\mathrm{np}}^{*}$ with respect to the separation $R$. In this respect we note that the nucleons inducing the NolenSchiffer anomaly are valence nucleons which, if the mirror nuclei differ by one particle or hole from the (magic) closed-shell case, must be located in the peripheral region of each of the mirror nuclei. For example, in a nuclear shell model with a Wood-Saxon potential the wave functions of the valence nucleon of a nucleus of mass number $A=A_{\text {magic }} \pm 1$, respectively, can be fitted very well by the Gaussian form

$$
\begin{aligned}
\psi_{A}^{(\mathrm{n}, \mathrm{p})}(R) & =\left(\frac{\left(b_{A}^{(\mathrm{n}, \mathrm{p})}\right)^{3}\left(b_{A}^{(\mathrm{n}, \mathrm{p})} R\right)^{2 n_{A}}}{2 \pi \Gamma\left(3 / 2+n_{A}\right)}\right)^{1 / 2} \\
& \times \exp \left\{-\frac{\left(b_{A}^{(\mathrm{n}, \mathrm{p})} R\right)^{2}}{2}\right\}
\end{aligned}
$$

where $\Gamma$ is the Gamma function and the parameters $b_{A}$ and $n_{A}$ have the following value: 8 :

$$
\begin{array}{lll}
b_{15}^{(\mathrm{p})}=0.871 m_{\pi}, & b_{15}^{(\mathrm{n})}=0.886 m_{\pi}, & n_{15}=1, \\
b_{17}^{(\mathrm{p})}=0.843 m_{\pi}, & b_{17}^{(\mathrm{n})}=0.860 m_{\pi}, & n_{17}=2, \\
b_{39}^{(\mathrm{p})}=0.785 m_{\pi}, & b_{39}^{(\mathrm{n})}=0.804 m_{\pi}, & n_{39}=2, \\
b_{41}^{(\mathrm{p})}=0.774 m_{\pi}, & b_{41}^{(\mathrm{n})}=0.794 m_{\pi}, & n_{41}=3 .
\end{array}
$$

The averaged neutron-proton-mass difference is given by 9

$$
\begin{aligned}
\Delta \bar{m}_{\mathrm{np}}^{*} & =\bar{m}_{\mathrm{n}}^{*}-\bar{m}_{\mathrm{p}}^{*}, \\
\bar{m}_{\mathrm{n}, \mathrm{p}}^{*} & \equiv \int m_{\mathrm{n}, \mathrm{p}}^{*}(R)\left(\psi^{(\mathrm{n}, \mathrm{p})}(R)\right)^{2} \mathrm{~d}^{3} R .
\end{aligned}
$$

Defining the difference of neutron-proton probability densities as

$$
\left(\psi^{(\mathrm{n})}(R)\right)^{2}-\left(\psi^{(\mathrm{p})}(R)\right)^{2} \equiv \Delta \psi_{\mathrm{np}}^{(2)}(R)
$$

one can rewrite eq. (53) as

$$
\begin{aligned}
\Delta \bar{m}_{\mathrm{np}}^{*} & \approx \int\left(\Delta \psi_{\mathrm{np}}^{(2)} m_{\mathrm{p}}^{*}+\left(\psi^{(p)}\right)^{2} \Delta m_{\mathrm{np}}^{*}\right) \mathrm{d}^{3} R \\
& \equiv \Delta \bar{m}_{\mathrm{np}}^{*(1)}+\Delta \bar{m}_{\mathrm{np}}^{*(2)},
\end{aligned}
$$

\footnotetext{
${ }^{8}$ For the values of the Wood-Saxon potential parameters see ref. 44.

9 Note that the wave functions $(\underline{52}$ ) are properly normalized.
}

ignoring the subleading contribution of the cross term $\int \Delta \psi_{\mathrm{np}}^{(2)} \Delta m_{\mathrm{np}}^{*} \mathrm{~d}^{3} R$.

Then the Nolen-Schiffer anomaly for a given pair of mirror nuclei with the mass number $A$ simply takes the form

$$
\bar{\Delta}_{\mathrm{NSA}}=\Delta m_{\mathrm{np}}-\left(\Delta \bar{m}_{\mathrm{np}}^{*(1)}+\Delta \bar{m}_{\mathrm{np}}^{*(2)}\right)
$$

The dependence of the Nolen-Schiffer anomaly on the mass number $A$ is presented in Table 2 (see the third column ' $\alpha_{\text {ren }}=0$, present approach'). One can see that our predictions of the behavior of the NSA qualitatively go into the right direction. But quantitatively, the results are too big by one order of magnitude. This is mainly due to the strong shift of $\Delta \bar{m}_{\mathrm{p}}^{*(1)}$ (see the third column of Table 2) that results for three reasons: (i) the rather large renormalization of the effective nucleon mass, (ii) the pronounced $R$ dependence of $m_{\mathrm{p}}^{*}$ inside the nucleus (see fig. 3), and (iii) the relative swelling of the proton distributions due to the Coulomb factor, i.e. $\Delta \psi_{\mathrm{np}}^{(2)} \neq 0$. For example, the averaged in-medium mass of the valence proton in ${ }^{17} \mathrm{O}$ is reduced to $\bar{m}_{\mathrm{p}}^{*}=812.35 \mathrm{MeV}$. This drop of about $125 \mathrm{MeV}$ is very large in comparison with the empirical value of the binding energy per nucleon in nuclear matter. For heavier nuclei, where the density in the interior approximates the normal nuclear matter density (see fig. 3), the drop of the averaged effective mass is even larger, e.g. $m_{\mathrm{p}}-\bar{m}_{\mathrm{p}}^{*} \sim 150-200 \mathrm{MeV}$ around ${ }^{40} \mathrm{Ca}$ (see the second column of Table 2) down to $\sim 300 \mathrm{MeV}$ around ${ }^{208} \mathrm{~Pb}$.

If only the contribution $\Delta \bar{m}_{\mathrm{np}}^{*(2)}$ (due to the explicit $R$ dependence of the neutron-proton mass difference) were considered, then the NSA in the present approach would even have a negative sign: $\Delta m_{\mathrm{np}}-\Delta \bar{m}_{\mathrm{np}}^{*(2)}<0$.

In our model the effective mass of the nucleon strongly depends on the phenomenological input parameters of the pion-nucleus sector, mainly via the $p$-wave scattering volume $c_{0}$ and the Landau parameter $g^{\prime}$ (see subsection[2.1). In ref. [45, according to the systematics of the effective axial coupling $g_{A}^{*}$ in finite nuclei of the range $5 \leq A \leq 39$ [4], the values $c_{0}=0.15 m_{\pi}^{-3}$ and $g^{\prime}=0.6$ were predicted 10 . These values imply a smaller drop of the effective mass of the nucleon, e.g. $m_{\mathrm{N}}^{*} / m_{\mathrm{N}} \sim 0.76$ in normal nuclear matter 45. Although, the use of smaller values of $c_{0}$ and bigger values of $g^{\prime}$ improves the NSA results (e.g., $\bar{\Delta}_{\mathrm{NSA}}=2.86 \mathrm{MeV}$ for the pair ${ }^{15} \mathrm{O}-{ }^{15} \mathrm{~N}$ for $c_{0}=0.15 m_{\pi}^{-3}$ and $\left.g^{\prime}=0.75\right)$, this is still not enough to satisfy the experimental data.

Instead of fine-tuning these parameters to unphysical values, one might rather invert the problem and try to estimate the effective nucleon mass inside finite nuclei according to the results in the isospin-breaking sector. To perform this task we fine-tune an artificially added renormalization parameter $\alpha_{\text {ren }}$ in the expression

$$
m_{\mathrm{n}, \mathrm{p}}^{*}\left(R, \alpha_{\mathrm{ren}}\right)=m_{\mathrm{n}, \mathrm{p}}^{*}(R)+\left(m_{\mathrm{n}, \mathrm{p}}-m_{\mathrm{n}, \mathrm{p}}^{*}(R)\right) \alpha_{\mathrm{ren}}
$$

10 Compare with the values $c_{0}=0.21 m_{\pi}^{-3}$ and $g^{\prime}=0.47$ used in the present work. 
Table 2. The averaged mass of the valence proton in a given nucleus $\bar{m}_{\mathrm{p}}^{*}$, the contributions to the effective neutron-proton mass difference (see eq. (55)) and the comparison of the Nolen-Schiffer discrepancy $\bar{\Delta}_{\mathrm{NSA}}$ (see eq.s (56), (57)) calculated in the present approach with other "empirical" results. All quantities are in units of MeV.

\begin{tabular}{|c|c|c|c|c|c|c|c|c|c|c|}
\hline \multirow{3}{*}{ Nuclei } & & & \multicolumn{6}{|c|}{ Present approach } & \multirow{3}{*}{$\begin{array}{l}\bar{\Delta}_{\mathrm{NSA}} \\
\text { ref. } 16\end{array}$} & \multirow{3}{*}{$\begin{array}{l}\bar{\Delta}_{\mathrm{NSA}} \\
\text { ref. } 17\end{array}$} \\
\hline & \multicolumn{2}{|c|}{$\bar{m}_{\mathrm{p}}^{*}$} & \multicolumn{3}{|c|}{$\alpha_{\text {ren }}=0$} & \multicolumn{3}{|c|}{$\alpha_{\text {ren }}=0.95$} & & \\
\hline & $\alpha_{\text {ren }}=0$ & $\alpha_{\mathrm{ren}}=0.95$ & $\Delta \bar{m}_{\mathrm{np}}^{*(1)}$ & $\Delta \bar{m}_{\mathrm{np}}^{*(2)}$ & $\bar{\Delta}_{\mathrm{NSA}}$ & $\Delta \bar{m}_{\mathrm{np}}^{*(1)}$ & $\Delta \bar{m}_{\mathrm{np}}^{*(2)}$ & $\bar{\Delta}_{\mathrm{NSA}}$ & & \\
\hline${ }^{15} \mathrm{O}-{ }^{15} \mathrm{~N}$ & 767.45 & 928.30 & -4.27 & 1.56 & 4.02 & -0.21 & 1.33 & 0.20 & - & $0.16 \pm 0.04$ \\
\hline${ }^{17} \mathrm{~F}-{ }^{17} \mathrm{O}$ & 812.35 & 930.54 & -5.53 & 1.52 & 5.33 & -0.28 & 1.32 & 0.27 & 0.31 & $0.31 \pm 0.04$ \\
\hline${ }^{39} \mathrm{Ca}^{-39} \mathrm{~K}$ & 724.78 & 926.16 & -8.11 & 1.67 & 7.75 & -0.41 & 1.33 & 0.37 & - & $0.22 \pm 0.08$ \\
\hline
\end{tabular}

of the effective nucleon mass in such a way that the NSA is satisfied. The results are presented in Table 2 (see the column ' $\alpha_{\text {ren }}=0.95$ '). It can be seen that a successful description of the correct order of the NSA implies a rather small drop of the mass of the valence nucleons: $m_{\mathrm{n}, \mathrm{p}}-\bar{m}_{\mathrm{n}, \mathrm{p}}^{*}\left(\alpha_{\mathrm{ren}}=0.95\right) \sim 10 \mathrm{MeV}$ which is close to the empirical binding energy per nucleon in nuclear matter. In this case the contribution to the NSA from $\Delta \bar{m}_{\mathrm{np}}^{*(2)}$ can be neglected: $\Delta m_{\mathrm{np}}-\Delta \bar{m}_{\mathrm{np}}^{*(2)}\left(\alpha_{\mathrm{ren}}=0.95\right) \sim-0.02 \mathrm{MeV}$.

\section{Conclusions and Outlook}

In summary, we have studied the effective neutron-proton mass difference $\Delta m_{\mathrm{np}}^{*}$ in finite nuclei in the framework of a medium-modified Skyrme model. The value of $\Delta m_{\mathrm{np}}^{*}$ for a given nucleus approaches an extremum near the nuclear surface where the local density gradients are large and the isospin asymmetry is high.

We have discussed the relevance of our results for the Nolen-Schiffer anomaly. Qualitatively, our approach predicts the correct behavior of the Nolen-Schiffer anomaly. But quantitatively it is not satisfactory: the results are one order of magnitude too large. Clearly, the part of our calculation relevant to the Nolen-Schiffer anomaly depends on the proton and neutron distributions of the mirror nuclei and is very sensitive to the behavior of the wave functions of the valence nucleons in the peripheral region of the nucleus. We have pointed out the possibility that the Nolen-Schiffer anomaly may rather follow from the behavior of the effective nucleon mass in finite nuclei than from the effective neutron-proton mass difference: our calculations imply that the Nolen-Schiffer anomaly could not and, maybe, should not be saturated by $\Delta \bar{m}_{\text {np }}^{*(2)}$ (the contribution due to the explicit density dependence of the neutron-proton mass difference). Rather more important is $\Delta \bar{m}_{\mathrm{np}}^{*(1)}$, the contribution due to the difference in the wave functions of valence proton and neutron weighted by the local (density and density-gradient induced) variation of the effective mass of the nucleon. In fact, when we restrict the in-medium reduction of the (averaged effective) proton mass to about $1 \%$ of the free proton mass, we obtain a rather precise description of the NSA.
Of course, the calculations of $\Delta E_{\mathrm{EM}}$ in ref. [17] (see eq. (51)) takes into account the effect due to the different wave functions of valence nucleons. But in ref. 17 a constant value of the nucleon mass was used in the calculations, namely the free mass. So it might be possible to resolve the anomaly of the mirror nuclei by considering a dynamical (local) mass of the nucleon that is only slightly reduced in comparison to the free mass. In this respect, it is interesting to note that in the local-densityfunctional approach to many-body nuclear systems an additional term has been introduced into the Coulomb part of the energy density of the nuclear system that removes the anomaly [49,50,51,52. Moreover, this term is chosen such that it is proportional to the isoscalar rather than the isovector density 12 . In this connection, it is even argued in refs. 53 ,54 that the effective-to-free-nucleon-mass ratio $m_{\mathrm{N}}^{*} / m_{\mathrm{N}}$ is unity to within a few percent. It seems that different model calculations lead to similar conclusions about the origin of the Nolen-Schiffer anomaly.

Returning to our model, we note that the results might be improved by a more self-consistent calculation that takes into account the feedback(s) between the modified skyrmion and the local nuclear background and by the inclusion of further degrees of freedom, which may anyhow be needed from more detailed considerations about the nucleon structure and the nucleon-nucleon interaction. Also the non-local character of the effective nucleon mass may be of importance. Note that many-body calculations (see e.g. 3, 4, 8, 9, 55]) indicate that, while $m^{*}$ is small inside the Fermi sphere, it can reach values close to the vacuum value near $k_{\mathrm{F}}$. In this connection, however, we should remark that the gradient terms which are present in our model do not noticeably affect the scaling behavior of $m^{*}$. They are important for the surface behavior of $\Delta m_{\mathrm{np}}^{*}$, though.

We would like to thank Horst Lenske for useful discussions and for a computer code calculating nuclear densities of finite nuclei which allowed us to extract the background-density parameters. We are also grateful to Frank Grümmer for provid-

\footnotetext{
11 This phenomenon is known as Thomas-Ehrman effect 47 48 .

12 Note, however, that the isoscalar contribution is surface dominated.
} 
ing us with calculated shell-model wave functions. The work of U.T.Y. was supported by the Alexander von Humboldt Foundation. The work of A.M.R. was supported by the second phase of the Brain Korea 21 Project in 2007. Partial financial support from the EU Integrated Infrastructure Initiative Hadron Physics Project (contract number RII3-CT-2004-506078), by the DFG (TR 16, "Subnuclear Structure of Matter") and by BMBF (research grant 06BN411) is gratefully acknowledged. This work is partially supported by the Helmholtz Association through funds provided to the virtual institute "Spin and strong QCD"(VH-VI-231).

\section{A Mass and moments of inertia of a classical soliton in the nonspherical scenario}

In the following, we use the definitions

$$
\begin{aligned}
& P_{r} \equiv \partial_{r} P, \quad P_{\theta} \equiv \partial_{\theta} P, \quad \Theta_{r} \equiv \partial_{r} \Theta, \quad \Theta_{\theta} \equiv \partial_{\theta} \Theta, \\
& S_{P} \equiv \sin P, \quad C_{P} \equiv \cos P, \quad S_{\Theta} \equiv \sin \Theta \\
& C_{\Theta} \equiv \cos \Theta, \quad S_{\theta} \equiv \sin \theta, \quad C_{\theta} \equiv \cos \theta .
\end{aligned}
$$

The mass of the nonperturbated system of the Lagrange functional (36) has the form

$$
\begin{aligned}
M_{\mathrm{NP}}^{*} & =\pi \int_{0}^{\infty} d r r^{2} \int_{0}^{\pi} S_{\theta} d \theta \times\left\{\frac{F_{\pi}^{2}}{4 r^{2}}\left(1-\chi_{p}^{0}(x)\right)\right. \\
& \times\left[P_{\theta}^{2}+r^{2} P_{r}^{2}+S_{P}^{2}\left(\frac{S_{\Theta}^{2}}{S_{\theta}^{2}}+\Theta_{\theta}^{2}+r^{2} \Theta_{r}^{2}\right)\right] \\
& +\frac{S_{P}^{2}}{e^{2} r^{4}}\left[\frac{S_{\Theta}^{2}}{S_{\theta}^{2}}\left(P_{\theta}^{2}+r^{2} P_{r}^{2}\right)\right. \\
& \left.+S_{P}^{2} \frac{S_{\Theta}^{2}}{S_{\theta}^{2}}\left(\Theta_{\theta}^{2}+r^{2} \Theta_{r}^{2}\right)+r^{2}\left(P_{r} \Theta_{\theta}-P_{\theta} \Theta_{r}\right)^{2}\right] \\
& \left.+\frac{m_{\pi}^{2} F_{\pi}^{2}}{2}\left(1+m_{\pi}^{-2} \chi_{s}^{00}(x)\right)\left(1-C_{P}\right)\right\},
\end{aligned}
$$

where $x=\sqrt{r^{2}+2 r R \cos \theta+R^{2}}$ 34. For the moment of inertia, we introduce the following definition

$$
\Lambda=2 \pi \int_{0}^{\infty} d r r^{2} \int_{0}^{\pi} S_{\theta} d \theta \lambda,
$$

where the contributions of the different parts of the Lagrange functional (36) are given as

$$
\begin{aligned}
\lambda_{\omega \omega, 12}^{*} & =\frac{F_{\pi}^{2}}{8}\left(1+\frac{\chi_{s}^{02}(x)}{m_{\pi}^{2}}\right)\left(1+C_{\Theta}^{2}\right) S_{P}^{2} \\
& +\frac{S_{P}^{2}}{2 e^{2} r^{2}}\left[\left(1+C_{\Theta}^{2}\right)\left(P_{\theta}^{2}+r^{2} P_{r}^{2}\right)\right. \\
& \left.+S_{P}^{2}\left(\frac{S_{\Theta}^{2}}{S_{\theta}^{2}}+C_{\Theta}^{2}\left(\Theta_{\theta}^{2}+r^{2} \Theta_{r}^{2}\right)\right)\right], \\
\lambda_{\omega \Omega, 12}^{*} & =\frac{F_{\pi}^{2}}{8}\left(1+\frac{\chi_{s}^{02}(x)}{m_{\pi}^{2}}\right)\left(C_{\theta} C_{\Theta} \frac{S_{\Theta}}{S_{\theta}}+\Theta_{\theta}\right) S_{P}^{2} \\
& +\frac{S_{P}^{2}}{2 e^{2} r^{2}}\left[C_{\theta} C_{\Theta} \frac{S_{\Theta}}{S_{\theta}}\left(P_{\theta}^{2}+r^{2} P_{r}^{2}+S_{P}^{2}\left(\Theta_{\theta}^{2}+r^{2} P_{r}^{2}\right)\right)\right.
\end{aligned}
$$

$$
\begin{aligned}
\lambda_{\Omega \Omega, 12}^{*} & =\frac{F_{\pi}^{2}}{8}\left(1+\frac{\chi_{s}^{02}(x)}{m_{\pi}^{2}}\right) \\
& \times\left[P_{\theta}^{2}+S_{P}^{2}\left(C_{\theta}^{2} \frac{S_{\Theta}^{2}}{S_{\theta}^{2}}+\Theta_{\theta}^{2}\right)\right] \\
& +\frac{S_{P}^{2}}{2 e^{2} r^{2}}\left[\frac { S _ { \Theta } ^ { 2 } } { S _ { \theta } ^ { 2 } } \left(\left(1+C_{\theta}^{2}\right)\left(P_{\theta}^{2}+S_{P}^{2} \Theta_{\theta}^{2}\right)\right.\right. \\
& \left.\left.+r^{2}\left(P_{r}^{2}+S_{P}^{2} \Theta_{r}^{2}\right) C_{\theta}^{2}\right)+r^{2}\left(P_{r} \Theta_{\theta}-P_{\theta} \Theta_{r}\right)^{2}\right],
\end{aligned}
$$$$
\lambda_{\omega \Omega, 33}^{*}=\frac{F_{\pi}^{2}}{4}\left(1+\frac{\chi_{s}^{02}(x)}{m_{\pi}^{2}}\right) S_{\Theta}^{2} S_{P}^{2}
$$$$
+\frac{S_{P}^{2}}{e^{2} r^{2}}\left(P_{\theta}^{2}+r^{2} P_{r}^{2}+S_{P}^{2}\left(\Theta_{\theta}^{2}+r^{2} \Theta_{r}^{2}\right)\right) S_{\Theta}^{2},
$$$$
\lambda_{\text {mes }}=\frac{F_{\pi}^{2}}{4} S_{\Theta}^{2} S_{P}^{2},
$$$$
\lambda_{\mathrm{env}}^{*}=\frac{F_{\pi}^{2}}{4} \Delta \alpha S_{\Theta}^{2} S_{P}^{2} .
$$

\section{B Charges and magnetic moments in the nonspherical scenario}

The zero component of the baryonic current in nonspherically deformed states has the form

$$
B_{0}=-\frac{S_{P}^{2}}{2 \pi^{2} r^{2}} \frac{\sin \Theta}{\sin \theta}\left(P_{r} \Theta_{\theta}-P_{\theta} \Theta_{r}\right) .
$$

In calculating the third component of the isospin current $V_{0}^{(3)}$, one finds

$$
\int \mathrm{d}^{3} r V_{0}^{(3)}=\left(a^{*}+\omega_{2}-\Omega_{3}\right) \Lambda_{\omega \Omega, 33}^{*}+\Lambda_{\mathrm{env}}^{*} \equiv T_{3}
$$

Consequently, the angle-averaged isospin charge density is given as

$$
\begin{aligned}
\tilde{T}_{3} & =\left(a^{*}+\omega_{2}-\Omega_{3}\right) \tilde{\lambda}_{\omega \Omega, 33}^{*}+\tilde{\lambda}_{\mathrm{env}}^{*} \\
& =\frac{T_{3}-\Lambda_{\mathrm{env}}^{*}}{\Lambda_{\omega \Omega, 33}^{*}} \tilde{\lambda}_{\omega \Omega, 33}^{*}+\tilde{\lambda}_{\mathrm{env}}^{*},
\end{aligned}
$$

where

$$
\begin{aligned}
\tilde{\lambda}_{\omega \Omega, 33}^{*} & =\frac{1}{2} \int_{0}^{\pi} \sin \theta \mathrm{d} \theta \lambda_{\omega \Omega, 33}^{*}, \\
\tilde{\lambda}_{\mathrm{env}}^{*} & =\frac{1}{2} \int_{0}^{\pi} \sin \theta \mathrm{d} \theta \lambda_{\mathrm{env}}^{*} .
\end{aligned}
$$

Since the charges of the nucleons are defined as

$$
Q^{\left(\begin{array}{c}
\mathrm{p} \\
\mathrm{n}
\end{array}\right)}=\frac{B}{2}+T_{3}^{\left(\begin{array}{l}
\mathrm{p} \\
\mathrm{n}
\end{array}\right)} \equiv \int \rho_{\mathrm{E}}^{\mathrm{S}}(r, \theta) \mathrm{d}^{3} r \pm \int \rho_{\mathrm{E}}^{\mathrm{V}}(r, \theta) \mathrm{d}^{3} r,
$$


the isoscalar and the isovector density distributions have here the following form

$$
\begin{aligned}
\rho_{\mathrm{E}}^{\mathrm{S}} & =\frac{B_{0}}{2}-\frac{\Lambda_{\mathrm{env}}^{*}}{\Lambda_{\omega \Omega, 33}^{*}} \tilde{\lambda}_{\omega \Omega, 33}^{*}+\tilde{\lambda}_{\mathrm{env}}^{*}, \\
\rho_{\mathrm{E}}^{\mathrm{V}} & =\frac{\tilde{\lambda}_{\omega \Omega, 33}^{*}}{2 \Lambda_{\omega \Omega, 33}^{*}} .
\end{aligned}
$$

Analogous calculations for the magnetic moments lead to

$$
\mu^{\left(\begin{array}{l}
\mathrm{p} \\
\mathrm{n}
\end{array}\right) *} \equiv \int \rho_{\mathrm{M}}^{\mathrm{S}}(r, \theta) \mathrm{d}^{3} r \pm \int \rho_{\mathrm{M}}^{\mathrm{V}}(r, \theta) \mathrm{d}^{3} r,
$$

where

$$
\begin{aligned}
\rho_{\mathrm{M}}^{\mathrm{S}} & =\frac{m_{\mathrm{N}}\left(1+\Lambda_{\mathrm{env}}^{*}\right)}{4 \Lambda_{\omega \Omega, 33}^{*}} B_{0} r^{2} \sin ^{2} \theta \\
\rho_{\mathrm{M}}^{\mathrm{V}} & =\frac{m_{\mathrm{N}}}{3}\left[\frac{F_{\pi}^{2}}{4}\left(1-\chi_{p}^{0}(x)\right) S_{P}^{2} S_{\Theta}^{2}+\frac{S_{P}^{2}}{e^{2} r^{2}}\right. \\
& \left.\times\left(P_{\theta}^{2}+r^{2} P_{r}^{2}+S_{P}^{2}\left(\Theta_{\theta}^{2}+r^{2} \Theta_{r}^{2}\right)\right) S_{\Theta}^{2}\right] .
\end{aligned}
$$

\section{References}

1. D. Lunney, J. M. Pearson, C. Thibault, Rev. Mod. Phys. 75, 1021 (2003).

2. I. Bombaci and U. Lombardo, Phys. Rev. C 44, 1892 (1991).

3. W. Zuo, I. Bombaci and U. Lombardo, Phys. Rev. C 60, 024605 (1999) arXiv:nucl-th/0102035.

4. W. Zuo, L. G. Cao, B. A. Li, U. Lombardo and C. W. Shen, Phys. Rev. C 72, 014005 (2005) arXiv:nucl-th/0506003.

5. E. Chabanat, J. Meyer, P. Bonche, R. Schaeffer and P. Haensel, Nucl. Phys. A 627, 710 (1997).

6. T. Lesinski, K. Bennaceur, T. Duguet and J. Meyer, Phys. Rev. C 74, 044315 (2006) arXiv:nucl-th/0607065.

7. M. Lopez-Quelle, S. Marcos, R. Niembro, A. Bouyssy and V. G. Nguyen, Nucl. Phys. A 483, 479 (1988).

8. E. N. E. van Dalen, C. Fuchs and A. Faessler, Phys. Rev. Lett. 95, 022302 (2005) arXiv:nucl-th/0502064.

9. E. N. E. van Dalen, C. Fuchs and A. Faessler, Eur. Phys. J. A 31, 29 (2007) arXiv:nucl-th/0612066.

10. S. Kubis and M. Kutschera, Phys. Lett. B 399, 191 (1997) arXiv:astro-ph/9703049.

11. B. Liu, V. Greco, V. Baran, M. Colonna and M. Di Toro, Phys. Rev. C 65, 045201 (2002) arXiv:nucl-th/0112034.

12. V. Greco, M. Colonna, M. Di Toro, G. Fabbri and F. Matera, Phys. Rev. C 64, 045203 (2001) arXiv:nucl-th/0011033.

13. F. Hofmann, C. M. Keil and H. Lenske, Phys. Rev. C 64, 034314 (2001) arXiv:nucl-th/0007050.

14. K. Tsushima, K. Saito and A. W. Thomas, Phys. Lett. B 465, 36 (1999) arXiv:nucl-th/9907101.

15. L. W. Chen, C. M. Ko, B. A. Li and G. C. Yong, arXiv:0704.2340 [nucl-th].

16. J. A. Nolen and J. P. Schiffer, Ann. Rev. Nucl. Part. Sci. 19, 471 (1969).

17. S. Shlomo, Rep. Prog. Phys. 41, 957 (1978).
18. B. A. Li, C. M. Ko and W. Bauer, Int. J. Mod. Phys. E 7, 147 (1998) arXiv:nucl-th/9707014.

19. V. Baran, M. Colonna, V. Greco and M. Di Toro, Phys. Rept. 410, 335 (2005) arXiv:nucl-th/0412060.

20. A. W. Steiner, M. Prakash, J. M. Lattimer and P. J. Ellis, Phys. Rept. 411, 325 (2005) arXiv:nucl-th/0410066.

21. S. Shlomo, Physica Scr. 26, 280 (1982).

22. E. M. Henley and G. Krein, Phys. Rev. Lett. 62, 2586 (1989).

23. T. Hatsuda, H. Hogaasen and M. Prakash, Phys. Rev. Lett. 66, 2851 (1991) [Erratum-ibid. 69, 1290 (1992)].

24. A. G. Williams and A. W. Thomas, Phys. Rev. C 33, 1070 (1986).

25. T. D. Cohen, R. J. Furnstahl and M. K. Banerjee, Phys. Rev. C 43, 357 (1991).

26. M. H. Shahnas, Phys. Rev. C 50, 2346 (1994).

27. C. J. Horowitz, J. Piekarewicz, Phys. Rev. C 63, 011303R (2000).

28. T. Suzuki, H. Sagawa and A. Arima, Nucl. Phys. A536, 141 (1992).

29. T. Schafer, V. Koch and G. E. Brown, Nucl. Phys. A 562, 644 (1993).

30. E. G. Drukarev and M. G. Ryskin, Nucl. Phys. A 572, 560 (1994).

31. C. Adami and G. E. Brown, Z. Phys. A 340, 93 (1991).

32. B. K. Agrawal, T. Sil, S. K. Samaddar, J. N. De and S. Shlomo, Phys. Rev. C 64, 024305 (2001).

33. U.-G. Meißner, A. M. Rakhimov, A. Wirzba and U. T. Yakhshiev, Eur. Phys. J. A 32, 299 (2007), arXiv:0705.1603 [nucl-th].

34. U. T. Yakhshiev, M. M. Musakhanov, A. M. Rakhimov, U.-G. Meißner and A. Wirzba, Nucl. Phys. A 700, 403 (2002) arXiv:nucl-th/0109008.

35. U. T. Yakhshiev, U.-G. Meißner and A. Wirzba, Eur. Phys. J. A 16, 569 (2003) arXiv:nucl-th/0211055.

36. W. M. Yao et al. [Particle Data Group], J. Phys. G 33, 1 (2006).

37. N. Kaiser and W. Weise, Phys. Lett. B 512, 283 (2001) arXiv:nucl-th/0102062.

38. E. E. Kolomeitsev, N. Kaiser and W. Weise, Phys. Rev. Lett. 90, 092501 (2003) arXiv:nucl-th/0207090.

39. R. A. Arndt, W. J. Briscoe, R. L. Workman and I. I. Strakovsky, the SAID $\pi N$ data base from http://gwdac.phys.gwu.edu/

40. A. Akhiezer, A. G. Sitenko, V. K. Tartakovski, Nuclear Elctrodynamics, Springer-Verlag, 1994.

41. F. Hofmann and H. Lenske, Phys. Rev. C 57 (1998) 2281 arXiv:nucl-th/9705049.

42. U.-G. Meißner, A. M. Rakhimov, A. Wirzba and U. T. Yakhshiev, Eur. Phys. J. A 31, 357 (2007) arXiv:nucl-th/0611066.

43. J. Gasser and H. Leutwyler, Phys. Rept. 87, 77 (1982).

44. V.A. Chepurnov, Yad. Fiz. 5, 955 (1967) [ Sov. J. Nucl. Phys. 6, 696 (1968)].

45. A. Rakhimov, M. M. Musakhanov, F. C. Khanna and U. T. Yakhshiev, Phys. Rev. C 58, 1738 (1998) arXiv:nucl-th/9609049.

46. B. Buck and S. M. Perez, Phys. Rev. Lett. 50, 1975 (1983).

47. R. G. Thomas, Phys. Rev. 81, 148 (1951).

48. J. B. Ehrman, Phys. Rev. 81, 412 (1951).

49. A. Bulgac, V. R. Shaginyan, Nucl. Phys. A 601, 103 (1996).

50. S. A. Fayans, JETP Lett. 68, 169 (1998). 
51. A. Bulgac, V. R. Shaginyan, Eur. Phys. J. A 5, 247 (1999).

52. S. A. Fayans and D. Zawischa, Int. J. Mod. Phys. B 15, 1684 (2001).

53. B. A. Brown, Phys. Rev. C 58, 220 (1998).

54. B. A. Brown, RIKEN Rev. 26, 53 (2000).

55. T. Frick, K. Gad, H. Müther and P. Czerski, Phys. Rev. C 65, 034321 (2002) arXiv:nucl-th/0111043. 